infarction, STEMI, diagnostic code) or their angiography was not identified as an emergency. Hospital data documented that 187 patients had CMR but, in the HES/PEDW data (inpatient and outpatient), CMR was recorded for only 53 of these (28\%). We are currently exploring algorithms to identify specific subgroups of particular interest with respect to CMR; early results suggest that we can identify patients who have had cardiac arrest or who have unobstructed arteries but not those with multivessel disease.

Conclusion It is possible to identify patients who activate the PPCI pathway from routinely collected HES/PEDW data. We conclude that it is not currently feasible to document the use of CMR in patients who activate the PPCI pathway from HES/PEDW data although the integration of the Diagnostic Imaging Dataset with inpatient and outpatient HES datasets may make it feasible soon. We are exploring the reasons for the discordance between CMR scans reported in the prospective cohort study and those identified in HES.

\section{P37 BARRIERS TO AND FACILITATORS OF EFFECTIVE DIABETES SELF-MANAGEMENT AMONG PEOPLE NEWLY DIAGNOSED WITH TYPE 2 DIABETES MELLITUS (T2DM): A QUALITATIVE STUDY FROM MALAYSIA}

${ }^{1} \mathrm{AM}$ Mohamed*, ${ }^{2} \mathrm{~J}$ Romli, ${ }^{1} \mathrm{~K}$ Ismail, ' $\mathrm{K}$ Winkley. 'Department of Psychological Medicine, Institute of Psychiatry, Psychology and Neuroscience, King's College London, London, UK; ${ }^{2}$ Non_Communicable Disease Unit, Primary Care Clinic Tampoi, Johor Bahru, Malaysia

\subsection{6/jech-2017-SSMAbstracts. 139}

Background To determine the views and experiences of people with Type 2 diabetes (T2DM) in relation to their diabetes self-management and to understand what additional support is required to support lifestyle changes.

Methods A qualitative study using semi-structured face-to-face and audio/telephone interviews. All interviews were audiorecorded, transcribed verbatim and analysed using a thematic approach. Seventeen people with newly diagnosed T2DM (less than 3 years of diagnosis) were recruited from a primary care clinic in the southern region of Malaysia (Johor Bahru).

Results Qualitative analysis revealed three major barriers to diabetes self-management: (i) psychological issues, e.g. depression and anxiety, such as feeling sad about the diagnosis and worried about the future; (ii) social factors e.g. shame and stigma of T2DM, feeling ashamed have diabetes at a young age and being different from peers ; (iii) perceived barriers e. g. environment and culture, such as ineffective support from healthcare providers, beliefs and use of herbal medicine, and the importance of eating rice and feast culture. Facilitators of diabetes self-management included greater perceived self-efficacy such as being disciplined about eating well, good support from immediate family members and religiosity.

Conclusion This study represents novel findings describing barriers and facilitators of effective T2DM self-management in Malaysia. It identifies specific cultural factors that are unique to the Malaysian population that have not been reported in western studies. If diabetes self-management education is to meet people's needs within this region factors such as these need to be considered when developing new T2DM education programmes.

\section{P38 DECISION REGRET IN MEN TREATED FOR LOCALISED PROSTATE CANCER: RESULTS FROM THE LIFE AFTER PROSTATE CANCER DIAGNOSIS STUDY}

${ }^{1} \mathrm{~A}$ Downing*, ${ }^{1} \mathrm{P}$ Wright, ${ }^{2} \mathrm{E}$ Watson, ${ }^{3} \mathrm{R}$ Wagland, ${ }^{4} \mathrm{~L}$ Hounsome, ${ }^{1} \mathrm{H}$ Butcher, ${ }^{5} \mathrm{~A}$ Gavin, ${ }^{1}$ AW Glaser. 'Leeds Institute of Cancer and Pathology, University of Leeds, Leeds, UK; ${ }^{2}$ Department of Applied Health and Professional Development, Oxford Brookes University, Oxford, UK; ${ }^{3}$ Faculty of Health Sciences, University of Southampton, Southampton, UKi ${ }^{4}$ National Cancer Registration and Analysis Service, Public Health England, London, UK; ${ }^{5}$ Northern Ireland Cancer Registry, Queens University Belfast, Belfast, UK

\subsection{6/jech-2017-SSMAbstracts. 140}

Background Men with localised prostate cancer have a number of treatment options. Treatments carry associated benefits and side- and late-effects. The Life After Prostate Cancer Diagnosis study is a UK-wide survey of men 18-42 months post-diagnosis of prostate cancer. The survey included treatment questions, the Decision Regret Scale (DRS) and a single item on decision-making participation. The aim of this sub-study is to explore the association of decision regret with prostate cancer treatment and patient perception of participation in decision making.

Methods The English arm of the survey achieved a $60.3 \%$ response rate (30 465 respondents). Men diagnosed with stage 1-3 disease were included in this sub-study $(n=16,808)$. Descriptive statistics were used to explore associations between DRS scores (0-100), self-reported treatments and perceived participation in decision-making.

Results 12600 (75.0\%) men completed the DRS. Due to the skewed nature of the data, decision regret was categorised as 'None' (score $=0 ; 36.5 \%)$, 'Low' (score $=5-20 ; 31.6 \%$ ) and 'High' (score $=\geq 25 ; 31.8 \%)$. Levels of regret were lowest in men who underwent brachytherapy alone and surgery alone (25.8\% and $27.5 \%$ respectively reporting 'High' regret). Men who received combination therapy (e.g. radiotherapy and hormones) reported higher levels of regret compared to men having a single therapy $(34.8 \%$ vs. $28.3 \%$ reporting 'High' regret). $28.6 \%$ of men on active surveillance reported a high level of regret. $74.2 \%$ of men said their views were definitely taken into account in treatment decisions: $22.6 \%$ of these reported high regret. $2.8 \%$ of men said their views were not taken into account; $62.8 \%$ reported high regret.

Conclusion These preliminary analyses show a strong association between perceived involvement in treatment decision making and subsequent decision regret in men with prostate cancer. Levels of regret also vary according to the type of treatment received. Interestingly, men on active surveillance who receive no treatment report similar levels of regret to those who undergo surgery. Further analysis will investigate the impact of patient characteristics and functional outcomes (urinary, bowel and sexual) on levels of regret.

\section{P39 EMERGENCY ADMISSIONS TO HOSPITAL FOR OLDER PEOPLE WITH COPD DURING THE RESHAPING CARE FOR OLDER PEOPLE PROGRAMME (RCOPP): AN ECOLOGICAL STUDY}

KA Levin*, EM Crighton. Public Health Directorate, NHS GGC, Glasgow, UK

10.1136/jech-2017-SSMAbstracts. 141 